\title{
Véronique GAZEAU, Monique GoULLET, Guillaume de Volpiano, un réformateur en son temps (962-1031)
}

\section{Bernard Merdrignac}

\section{(2) OpenEdition}

\section{Journals}

Édition électronique

URL : http://journals.openedition.org/abpo/520

DOI : 10.4000/abpo.520

ISBN : 978-2-7535-1515-4

ISSN : 2108-6443

Éditeur

Presses universitaires de Rennes

Édition imprimée

Date de publication : 30 octobre 2009

Pagination : 222-224

ISBN : 978-2-7535-1008-1

ISSN : 0399-0826

Référence électronique

Bernard Merdrignac, « Véronique Gazeau, Monique Goullet, Guillaume de Volpiano, un réformateur en son temps (962-1037)», Annales de Bretagne et des Pays de l'Ouest [En ligne], 116-3 | 2009, mis en ligne le 30 octobre 2009, consulté le 23 septembre 2020. URL : http://journals.openedition.org/abpo/520 ; DOI : https://doi.org/10.4000/abpo.520

Ce document a été généré automatiquement le 23 septembre 2020

(c) Presses universitaires de Rennes 


\title{
Véronique GAZEAU, Monique GOULLET, Guillaume de Volpiano, un réformateur en son temps (962-1031)
}

\author{
Bernard Merdrignac
}

\section{RÉFÉRENCE}

«Vita domni Willelmi » de Raoul Glaber, Texte, traduction, commentaire, Caen, Publications du CRAHM, 2008

1 L'inscription d'une question d'histoire médiévale intitulée « Pouvoirs, Église et société dans les royaumes de France, Bourgogne et Germanie aux $\mathrm{x}^{\mathrm{e}}$ et $\mathrm{xl}^{\mathrm{e}}$ siècles " au programme des concours de recrutement de l'enseignement secondaire a bien entendu été, comme d'habitude, l'occasion de la publication de manuels de qualité inégale et a donné lieu à la parution d'excellents essais historiques ${ }^{1}$. L'un de ses effets collatéraux dont il faut se féliciter est l'édition de cette «Vie du seigneur abbé Guillaume, premier abbé de Fécamp ", grâce à la collaboration entre Véronique Gazeau, professeure à l'université de Caen et membre du «Centre de recherches archéologiques et historiques médiévales » (UMR 6577 CNRS, CRAHM) et Monique Goullet, directrice du «Laboratoire de médiévistique occidentale de Paris » (UMR 8589 CNRS, LAMOP - université Paris 1Panthéon Sorbonne). Cette Vie latine n'avait en effet jamais bénéficié d'une traduction française. On sait que Georges Duby a largement contribué à asseoir parmi les historiens actuels la réputation de Raoul Glaber ( $†$ 1047, au plus tard), mais jusqu'à présent, ce sont surtout les cinq livres de ses Histoires qui ont retenu l'attention de ceux-ci, notamment grâce à la traduction récente de Mathieu Arnoux (Brepols, Turnhout, 1996). Outre qu'elles comportent de pittoresques anecdotes qui sont une aubaine pour les éditeurs de recueils de sources médiévales, les Histoires de Raoul Glaber, composées entre 1031 et 1047, passent pour représentatives de l'« historiographie proprement clunisienne » qui s'épanouit sous l'abbatiat prestigieux 
d'Odilon de Mercœur († 1049) à qui elles sont dédiées. Raoul précise par ailleurs qu'il a entrepris cet ouvrage à l'instigation de Guillaume de Volpiano ( $†$ 1031) à qui il a pu servir de secrétaire à un moment de sa carrière mouvementée de moine gyrovague. Selon son propre témoignage, c'est pendant la rédaction de cette œuvre monumentale (peut-être durant un séjour à Cluny) qu'une apparition de Guillaume avec qui il s'était brouillé (1028) l'aurait déterminé à écrire la biographiede celui-ci. Cela n'implique pas pour autant que ce dernier était déjà décédé dans la mesure où il n'était pas exceptionnel alors de composer une Vita du vivant de son héros. Les auteurs évoquent (p. 7, n. 20) les exemples contemporains des Vitae de Jean de Gorze († 974) ou de Léon IX († 1054). Sans doute pourrait-on même remonter au précédent de la Vita Martini, tête de série de l'hagiographie latine, entreprise par Sulpice Sévère avant la mort de saint Martin ( $† 397)$ ? Cela permet en tout état de cause de proposer la fourchette 1028-1031 pour la rédaction de ce document.

2 Jusqu'en 1974, le texte de la Vita n'était pratiquement accessible que dans des éditions du XVII ${ }^{e}$ siècle à partir d'un manuscrit (perdu) de l'abbaye Saint-Bénigne de Dijon, réformée par Guillaume qui en a été l'abbé de 990 à sa mort. La mise au jour, voici 35 ans, par Neithard Bulst d'une copie du $3^{e}$ quart du xie siècle (Paris BnF lat. 5390) associée dans le manuscrit au Traité sut l'Antéchrist d'Adson († 992) de Montier-en-Der (qui fut peut-être abbé de Saint-Bénigne quelque temps avant Guillaume) et à la Prophétie de la Sybille, incite à supposer que Raoul Glaber avait réuni pour écrire ses Histoires un dossier influencé par «les idées eschatologiques ». "À moins qu'il faille plutôt y voir une sélection voulue par le commanditaire » de cette copie de la Vita, ajoutent les auteurs (p.20). Neithard Bulst a identifié ce dernier, selon toute probabilité, avec Jean de Fécamp ( $†$ 1078), abbé de Saint-Bénigne pendant quelques années (1052-1054), et surtout successeur de Guillaume à la tête de l'abbaye de Fécamp que celui-ci venait de réformer. En toute hypothèse, le texte latin édité par N. Bulst (et repris ici avec quelques légères corrections) est donc proche de l'autographe. La traduction de M. Goullet, spécialiste de latin médiéval, serre le texte au plus près en préservant les exigences minimales de lisibilité et d'élégance conformes aux usages universitaires. La "présentation littéraire " et le "commentaire historique » qui encadrent cette édition soigneusement annotée sont bienvenus pour faciliter l'accès à un document qui illustre les enjeux du mouvement de "restauration-innovation » monastique de la fin $d u x^{e}$ siècle et des premières décennies $d u \mathrm{XI}^{\mathrm{e}}$ siècle (pour reprendre l'expression que $\mathrm{M}$. Soria Audebert et $\mathrm{C}$. Treffort préfèrent, pour leur part, au terme passe-partout de "réforme»). Enfin une bibliographie, à jour des acquis récents de la recherche internationale sur la question, permettrait au besoin d'approfondir celle-ci. Pour être complet, on pourrait toutefois y adjoindre les Actes de la journée d'étude du millénaire de Fruttuaria ${ }^{2}$.

3 Les relations entre " histoire et hagiographie » sont abordées succinctement dans un paragraphe (p. 8-9) destiné surtout à évaluer la « valeur historique » du témoignage de Raoul Glaber. Dans la perspective des concours qui supposent que les candidats aient conscience de la diversité et de la spécificité des sources historiques, il n'est sans doute pas inutile de rappeler qu'un "certain nombre de lieux communs" constituent des "figures imposées» de l'hagiographie. On évitera toutefois de prendre à la lettre l'assertion selon laquelle «les grands auteurs sont souvent de piètres hagiographes " qui conduirait presque à appliquer anachroniquement la célèbre formule d'André Gide sur « bons sentiments » et « mauvaise littérature » à Baudri de Bourgueil († 1130) dont 
est dénoncée (trop catégoriquement sans doute ici ?) la "platitude affligeante » des œuvres hagiographiques. Dans la mesure où la carrière de Robert d'Arbrissel figure aussi au programme des concours, les lecteurs des $A B P O$ auraient plutôt intérêt à consulter la note critique de Monique Goullet sur la récente édition par Jacques Dalarun et son équipe de la Vie de Robert composée par Baudri ${ }^{3}$. Ce n'est pas « rivé, contraint et forcé, à sa table de travail " que ce dernier a rédigé des Vies de saints. Son statut d'«hagiographe professionnel» relève au contraire à ses yeux de sa charge d'archevêque de Dol (1107-1130) comme l'a démontré la thèse soutenue en 2006 par $\mathrm{M}^{\text {me }}$ Armelle Le Huérou devant l'université de Rennes 2. Jean-Yves Tilliette, spécialiste de l'œuvre poétique de Baudri, se fonde sur cette thèse (sous presse) pour avancer que l'échec de Baudri à faire de Robert autre chose qu'un «ectoplasme ou un portrait robot" est assumé par cet auteur qui conclut la VitaRoberti sur le topos de l'« ineffable», en constatant qu'il n'a «presque rien dit» (tamen nihil diximus)! Si la " machine tourne à vide » c'est, d'après Jean-Yves Tilliette, parce que l'hagiographe n'a guère d'affinité avec son héros. «Bénédictin conservateur » (on serait tenté de nuancer en le qualifiant plutôt de réformateur timoré), Baudri «n'a pas compris la spiritualité de Robert et a même condamné ce qu'il en comprenait ». Au contraire, Raoul Glaber n'est sans doute pas « un grand auteur » et c'est d'ailleurs probablement leur caractère "anticonformiste " par rapport aux critères de son époque qui rendent ses écrits séduisants « au moins selon le goût actuel».

Le "commentaire historique " qui occupe un bon tiers de ce petit livre dépasse le simple attrait de la lecture d'un texte assez vivant qui associe « récit biographique » et « discours hagiographique ». Il permet, comme il se doit, de cerner la personnalité et la carrière d'un des abbés "réformateurs" les plus importants de son époque. «Volpiano » (un domaine familial rattaché à sa fondation piémontaise de Fruttuaria), « Dijon » (où Maieul de Cluny [†994] le place à l'abbatiat de Saint-Bénigne), « Fécamp » (que le duc Richard II de Normandie [ $†$ 1026] l'invite à restaurer), ce triple surnom qui s'attache à Guillaume atteste de l'échelle à laquelle s'exerce son action. Ses abbatiats multiples, ses allers-retours entre l'Italie et la Bourgogne, ses déplacements en Lotharingie, en Île-de-France puis en Normandie sont pour lui l'occasion de mettre en action une discretio toute clunisienne afin de soutenir son œuvre réformatrice dans ses rapports avec l'épiscopat ou les pouvoirs princiers et ses relations indirectes avec le pouvoir impérial. Guillaume n'a laissé aucun écrit. C'est pourquoi le sermon de la consécration de l'abbatiale Saint-Bénigne (30 octobre 1016) - probablement réécrit par Raoul Glaber pour en faire le "morceau de bravoure" de la Vita (c. 12) - fixe définitivement la figure du réformateur tel que le conçoit celui-ci : «un pédagogue austère et intransigeant, un donneur de leçons salutaires, un saint aux multiples visages. En s'adressant aux laïques, mais aussi aux évêques consécrateurs présents, il exalte le monachisme». Les quelques pages très denses de commentaire qu'y consacrent les auteurs (p. 116-118) sont aussi un modèle du genre susceptible de rendre de grands services aux premiers des utilisateurs du présent ouvrage qui seront, n'en doutons pas, les étudiants préparant les concours (et leurs enseignants). 


\section{NOTES DE FIN}

1. . À recommander particulièrement (et pas seulement aux candidats aux concours) : Soria-Audebert, M.,Treffort, C., Pouvoirs, Église, société. Conflits d'intérêts et convergence sacrée (IX ${ }^{e}$-XI ${ }^{e}$ siècle), Rennes, PUR, 2009.

2. . LucionI, A. (dir.), Guglielmo da Volpiano, la persona et l'opera, Atti della giornata di studio San Benigno Canavese, 4 ottobre 2003, Cantalupa, Effarà, 2005.

3. . GOULLET, M., « Robert d'Arbrissel entre la philologie et l'histoire », Revue Mabillon, t. 79 [2007] p. 275-278).Cf.DALARUN, J., GIORDANENGO, G., LE HUËROU, A., LONGÈRE, J., POIREL, D.,

VENARDE, B.-L.,Les Deux Vies de Robert d'Arbrissel, fondateur de Fontevraud. Légendes, écrits et témoignages. The two Lives of Robert of Arbrissel, founder of Fontevraud. Legends, writings, and testimonies, Brepols, Turnhout, 2006.

\section{AUTEURS}

BERNARD MERDRIGNAC 